\title{
An Ethical Overview of the CRISPR-Based Elimination of Anopheles gambiae to Combat Malaria
}

\author{
India Jane Wise (iD) Pascal Borry
}

Received: 13 August 2021 / Accepted: 2 December 2021 /Published online: 17 February 2022

(C) Journal of Bioethical Inquiry Pty Ltd. 2022

\begin{abstract}
Approximately a quarter of a billion people around the world suffer from malaria each year. Most cases are located in sub-Saharan Africa where Anopheles gambiae mosquitoes are the principal vectors of this public health problem. With the use of CRISPR-based gene drives, the population of mosquitoes can be modified, eventually causing their extinction. First, we discuss the moral status of the organism and argue that using genetically modified mosquitoes to combat malaria should not be abandoned based on some moral value of A. gambiae. Secondly, we argue that environmental impact studies should be performed to obtain an accurate account of the possible effects of a potential eradication of the organism. However, the risks from the purposeful extinction of A. gambiae should not overtake the benefits of eradicating malaria and risk assessments should be used to determine acceptable risks. Thirdly, we argue that the eventual release of the genetically modified mosquitoes will depend on transparency, community involvement, and cooperation between different nations.
\end{abstract}

Keywords Bioethics · Biodiversity - Gene drives · CRISPR-Cas9 $\cdot$ Malaria $\cdot$ Public health

I. J. Wise $(\bowtie) \cdot$ P. Borry

Centre for Biomedical Ethics and Law (CBMER), Department of Public Health and Primary Care, Faculty of Medicine, KU Leuven, Kapucijnenvoer 35 Box, 70013000 Leuven, Belgium e-mail: indiajanewise@gmail.com

P. Borry

e-mail: pascal.borry@kuleuven.be

\section{Introduction}

Malaria poses a significant public health threat around the world. According to the World Health Organization (WHO) Malaria Report (2020c), in 2019, there were 229 million malaria cases worldwide with a death toll of 409,000. These numbers do not include asymptomatic malarial infections which can also have a significant impact on the health of infected individuals (Chen et al. 2016). Infections are only expected to increase with global warming (Caminade et al. 2014). Malaria is caused by the parasite Plasmodium which replicates in, and is transmitted through, female Anopheles (Ancient Greek for "useless") mosquitoes - the vectors. Out of the five Plasmodium species that cause malaria in humans, Plasmodium falciparum is responsible for the highest death rate, while Plasmodium vivax causes the most malaria cases. There are around 515 Anopheles species, of which around thirty to forty are deemed important malaria vectors (World Health Organization 2017, 2020a).

Until the twentieth century, malaria was common in more than half the world. Only since then have humans made progress in battling the disease. Malaria was endemic in Europe until the 1970s, with Macedonia being the last country to eliminate the disease in 1975 (Piperaki 2018). By 2002, malaria was reduced to presence in 27 per cent of the global land surface (Hay et al. 2004). This indicates that malaria is not inherently a tropical disease but rather a disease that has been eliminated everywhere but the tropics. Today, malaria is endemic in eighty-seven countries, with the WHO 
African region carrying around 94 per cent of global malaria cases and deaths (World Health Organization 2020a).

A vital part of public health interventions against malaria has been vector control, since eliminating the Plasmodium parasite in isolation has proven to be difficult. Plasmodium parasites that cause malaria are very complex organisms with many different life and multiplication stages both in mosquitoes and humans. A lot of effort has been put into the development of vaccines that target the parasite, with the recent RTS,S vaccine being the first released antimalarial vaccine, but a vaccine with high efficacy and which provides protection for an extended period of time is not likely to become available soon (Feachem et al. 2019; World Health Organization 2021). The decrease in malaria accomplished so far, has therefore been due to major public health efforts focused on prevention through the use of insecticides, reduction in breeding sites for mosquitoes, and socioeconomic development (Roser and Ritchie 2019). Nevertheless, malaria rates have not been significantly reduced in recent years, one of the reasons being the development of insecticide-resistant mosquitoes (World Health Organization 2019a, 2020c). Progress has been made, but complete eradication is far from sight. Therefore, researchers have been pursuing other possibilities.

In 2014, a new technology was introduced that combines gene drives with CRISPR-Cas9. A gene drive is a genetic modification that allows certain genes to spread throughout the population by increasing their inheritance rate. Normally, a heterozygous genetic modification is inherited in 50 per cent of offspring. When combined with CRISPR-Cas9, however, the genetic modification is also copied to the homologous chromosome, resulting in the homozygous presence of this modification. This means the mutation will be present in both copies of the chromosome pair. This way, the mutation is inherited in 100 per cent of offspring, in which it is again copied to the homologous chromosome and the altered genes spread even faster (Esvelt et al. 2014; Hayirli and Martelli 2019; Scudellari 2019). In 2018, Kyrou et al. developed a CRISPR-based gene drive that involves the doublesex gene. The technique was focused on the Anopheles gambiae mosquito, which is a vector of the $P$. falciparum parasite. When this gene is disabled in female mosquitoes, they develop both male and female organs. Their proboscis turns male, making them unable to pierce human skin and they become infertile. This technique focuses on female
Anopheles since they, in addition to the flower nectar all Anopheles feed on, require blood to obtain protein for the egg-laying process. Male Anopheles are unable to pierce skin and transfer Plasmodium. When the doublesex genes were introduced in a caged population of Anopheles species, the population died out after seven to eleven generations (Kyrou et al. 2018). Were these genetically modified mosquitoes (GMMs) to be introduced into the wild, this could lead to the eradication of the entire targeted species. Therefore, the technique has a good chance of contributing to malaria eradication, given the difficulty for the parasite to find a new vector organism (Callaway, 2015; Hammond et al., 2017).

Due to the adverse effects on the environment this technology might have, the use of gene drives has been met with great criticism (Brossard et al. 2019). Direct effects through the eradication of a species or indirect effects through crossbreeding might lead to unexpected consequences for human and environmental health. Possibilities include changes in ecosystem dynamics or the spreading of genes to other species (Neves and Druml 2017). So far, the technique has only been used in a controlled environment, but modified mosquitoes might soon be released in nature. The Bill and Melinda Gates Foundation have invested in finding a gene drive solution and Bill Gates expects to have regulatory approval by 2024 and to have the first gene drive mosquitoes ready by 2026 (Gates 2019). Before this happens, the ethical implications should be thoroughly examined.

In this paper, various ethical concerns that arise regarding the eradication of a malaria species through gene-drive based genetic engineering will be discussed. The discussion will be related to: (a) the value of a species, (b) the environmental impact due to both the eradication of A. gambiae and the use of gene drives, and (c) the importance of community involvement. This will illuminate complexities and contribute to making a well-informed decision on the implementation of such a measure.

\section{The Value of a Species}

Biological diversity or biodiversity is traditionally defined as "the variety of life, in all its many manifestations" (Sodhi and Ehrlich 2010, 41). It has various stages and includes the variety of genes, species, populations, and entire ecosystems (Carrington 2019). As described above, the proposed technique could lead to 
the eradication of a species and thus a reduction in biodiversity. The conservation of biodiversity, however, is an objective of many organizations and governments (World Wildlife Fund (WWF) 2015; European Commission 2017; IUCN 2020; UN 2020). Biodiversity is essential to numerous benefits for the world including the provision of food, purification of water, stabilization of the earth's climate, and aesthetic advantages, among many others (WWF 2015). Biodiversity is perceived to be of major value to the world, but does this mean that all species have value and should be protected?

It is clear that not all organisms are treated equally. Organisms include every life form, while animals are specifically multicellular organisms which are not plants or fungi. Unicellular organisms are usually not included in life protection programmes, even though they exhibit high extinction rates (Weinbauer and Rassoulzadegan 2007; Louca et al. 2018). The eradication of viruses, which are part of the grey area between living and nonliving, has historically been seen as a triumph: the variola virus that causes smallpox has been eradicated since 1980, thanks to great public health efforts. This has been tremendously beneficial for humankind and, rightly, has been celebrated (CDC 2016). Eradication of the recent SARS-CoV-2 would surely also be praised globally. The Guinea worm parasite has been at the centre of a global eradication programme since 1980 . With only twenty-eight cases reported in 2018, the Guinea worm is likely to be the second deliberately eradicated species for public health purposes (CDC 2019). However, eliminating the parasite that causes malaria is not yet an option. Malaria prevention through the targeting of mosquitoes has been the main focus of interest instead. However, the importance of the conservation of insects, and more specifically mosquitoes, has not yet been defined clearly. Within the animal kingdom, the so called "higher species"- - vertebrates other than fish-have been the focus of conservation programmes (CITES 2011; World Animal Protection 2018; Pandas International 2020; Save the Rhino 2020). The question then follows where to draw the line between morally acceptable and non-acceptable extinction of a species.

Intuitively, the idea that all organisms or animals are equal, independent of their species, is not the common perception of most of the world's population. The public reaction towards a species' eradication depends mainly on the moral status of the organism as well as the possible consequences that could accompany its eradication (Pugh 2016). When a study in Sweden asked citizens about the use of insecticides against mosquitoes, many answered that they did not want to eliminate the entire species because that would feel morally wrong (Ojala and Lidskog 2011). However, in this case, this mosquito species only caused minor nuisances and not hundreds of thousands of deaths.

Mosquitoes as a species are generally ascribed a low or even no moral status (Anopheles meaning useless in Greek does not help its case). There also is a difference between killing some mosquitoes and purposefully eradicating an entire species of mosquitoes. Moral status is not a black and white question but one of degree (DeGrazia 2008). David DeGrazia distinguishes a twotier model from a sliding-scale model, as accounts of ascribing moral status. In this two-tier model, people are ascribed moral status while other sentient organisms have a lower moral status but never no moral status at all. Using the sliding-scale model, the amount of moral status an organism is afforded, depends on their degree of cognitive, affective, and social complexity. Using this model, humans are attributed the highest degree of moral status and non-sentient beings the lowest. In the same line, Peter Singer classifies organisms in different categories depending on sentience and self-awareness. Sentient and self-aware organisms deserve full moral consideration whereas insentient organisms do not (BBC 2014).

A different stance is taken by those who believe that all species have intrinsic value. According to biologist Michael Soulé $(1985,731)$ "Species have value in themselves, a value neither conferred nor revocable, but springing from a species' long evolutionary heritage and potential." This idea is defended by many other conservationists and equivalent with Tom Regan's philosophy that all species have intrinsic value because they are subjects-of-a-life (Regan 2004). However, Regan talks exclusively about animals, not organisms. It is unclear whether insects can also be seen as subjects-of-a-life because it is unclear to what extent they have some level of consciousness or even if they can feel (some) pain. Even if some insects were somewhat sentient, and the evidence points in the opposite direction, does this mean that they should be protected? Singer himself thinks that, regardless of their sentience, a campaign for insect rights is not yet on the agenda (Singer 2016). So, based on Singer's and Regan's views, fighting for mosquito rights is not in order. 
As to the quantitative measure of biodiversity, it should be considered that malaria is only caused by around thirty to forty Anopheles species. As there are over 3500 mosquito species, eradicating thirty of those Anopheles species, would only reduce the biodiversity of all mosquitoes by one percent (AMCA 2019). Moreover, the species in which the doublesex genes were disabled in 2018, were A. gambiae mosquitoes. Specifically eradicating this species would barely make an impact on the total biodiversity of mosquitoes.

Another topic of concern could be that if some species are protected and some are intentionally eradicated, this might lead to programmes to eradicate any number of species: a slippery slope. Indeed, eradicating one mosquito species might provide precedent to justify the eradication of other mosquito species. Focusing on the eradication of mosquito species that transmit dengue, yellow fever, or Zika is a logical next step if the eradication of A. gambiae is successful.

Considering the moral status ladder and the views on mosquitoes as non-sentient beings, discussed in the previous paragraphs, it seems that sacrificing mosquitoes for human lives is morally permissible. However, the function of a species must always be taken into account and other possibilities to eradicate these diseases must be considered. Eradication of a species is only permissible if it saves a considerable number of human lives or protects the ecosystem, if there is no other way. Eradication should never be the first option but rather the very last.

\section{Environmental Impact}

Disapproval of the use of gene drives has mainly been caused by fear of harming the environment, either through the eradication of a species or through the possible threats gene drives themselves can pose to the environment. These issues will be discussed in the following sections.

\section{Impact of the Elimination of Anopheles gambiae}

Besides being a source of food for other species, insects, including mosquitoes, are responsible for the pollination of the majority of food crops and plant species. These in turn provide food for many animal species (van der Sluijs and Vaage 2016). However, in this study, the focus lies on the elimination of Anopheles gambiae.
Therefore, solely the role of these Anopheles species within their native ecosystem needs to be investigated.

The impact of the elimination of a species can be determined through the losses it causes (Gascon et al. 2015). Not much is known about the pollination effects of A. gambiae mosquitoes, but studies indicate that A. gambiae do not occupy an essential role in the food cycle (Collins et al. 2018). However, losses can sometimes be hard to determine due to complex interactions in the ecosystem (Gascon et al. 2015). In general, high biodiversity is an indicator of a healthy ecosystem and a stable environment (European Commission 2019). This relates to the concept of redundancy, to what extent other species can take over functions in a system if other species were to disappear (Rosenfeld 2002). For the case of A. gambiae elimination, this means that its disappearance would reduce the biodiversity and redundancy in the ecosystem. However, redundancy in the ecosystem would most likely mean that the mosquito species' functions would be taken over, neutralizing the harmful effect of its extinction. The majority of experts agree that the effects on the local ecosystem of a species of mosquito disappearing, would not be significant (Fang 2010). Before genetically engineered mosquitoes can be released, field trials have to be performed in order to provide a thorough overview of the ecological dependency on A. gambiae mosquitoes. Recently, Target Malaria, a not-for-profit organization funded through the Bill \& Melinda Gates Foundation that focusses on finding gene drive solutions against malaria, has received a grant for a four-year study from Open Philanthropy, a research foundation. Amongst other things, the study will investigate potential ecological effects of the disappearance of A. gambiae (Open Philanthropy 2017; Target Malaria 2020).

These and other environmental impact studies will determine the impact of releasing genetically engineered mosquitoes into the wild.

\section{The Impact of Gene Drives}

The use of CRISPR-based gene drives has been met with controversy, due to its self-replicating and potentially irreversible nature (Cisnetto and Barlow 2020). However, the current measures taken against malaria should be considered in order to make accurate assessments of the effects of gene drives. If current measures against malaria are associated with greater adverse effects than the elimination of $A$. gambiae species or the 
use of gene drives, the development of gene drive technologies should be encouraged.

The two main forms of vector control today are the use of insecticide-treated mosquito nets and indoor spraying with residual insecticides (World Health Organization 2020a). The insecticides that are used do not discriminate between the different mosquito species and also target species that do not transmit human diseases (Hammond and Galizi 2017). On the other hand, they also target mosquitoes that transmit other diseases. Gene drives are targeted against one specific species and can therefore be regarded as a technology which has a reduced negative impact on biodiversity. However, this advantage would disappear if crossbreeding or horizontal gene transfer occurred between the genetically modified A. gambiae and related mosquito species. To prevent this, control strategies through molecular constraints have to be implemented that eliminate the threat of the gene drives spreading to non-target species (Naegeli et al. 2020).

A common argument against CRISPR-based gene drives is that they cause irreversible changes in genes. However, insecticide-resistance that is being built up by Anopheles species also makes inheritable changes in their genes with possible environmental impacts (Nkya et al. 2013). Then again, resistance to the gene drives can also be developed by the genetically modified mosquitoes (GMMs) which might cause environmental harm (Unckless, Clark, and Messer 2017). Insecticides would most likely still have to be used to protect against other mosquito-borne diseases such as dengue, Zika, or yellow fever, but were GMMs released into the environment, this would most likely lead to a decreased dependency on these insecticides (Hammond and Galizi 2017). Moreover, new genetic elements might reverse gene drives, disputing the irreversibility of the technology (Xu et al. 2020). In conclusion, as the technology improves continuously, some current arguments against gene drives will no longer be relevant.

Maximizing benefits and minimizing risks in public health interventions is not an easy feat. No intervention has zero risks, so a risk-weighing principle has to be applied. Possible environmental risks and public health benefits, being a significant decrease in the spread of malaria, must be balanced against each other. The question arises whether it is acceptable to promote public health at the expense of the environment. It should also be taken into account that when the environment is harmed, often public health also suffers. A decision then must be made about how much impact would be permissible, before it is deemed unethical to proceed. In other words: to what additional unwanted effects should we consent to achieve malaria eradication? The WHO recommends a risk assessment for the release of GMMs in their guidance framework for testing of genetically modified mosquitoes. It is pointed out that in countries with national environmental policies and protection goals, these should determine the threshold of what are acceptable risks. A general standard that could be used is also mentioned: "whether a specific GMM implementation 'causes more harm' than populations of wild mosquitoes managed under current practice" (World Health Organization 2014, 40). This standard has previously been used in Australia for the release of Aedes aegypti mosquitoes containing the Wolbachia bacteria in order to combat dengue (Murphy and Jansen 2010).

Another principle of risk mitigation that can be used is the precautionary principle. The precautionary principle emphasizes preventive decision-making in order to protect public health or the environment when there is a plausible risk (European Commission 2000; World Health Organization 2014). It promotes preventive rather than reactive action. The onus is on the proponents of the measure, meaning that they have to prove that the technology is safe rather than other parties having to prove that it is unsafe (Pearce 2004). The Convention on Biological Diversity highlights the importance of the precautionary approach when there is a threat to biological diversity caused by products of synthetic biology (Convention on Biological Diversity Conference of the Parties 2012). However, a strict interpretation of the principle implies that "regulatory approval should not be granted until all possible or theoretical risk and safety issues are scientifically resolved, regardless of societal needs and potential benefits" (World Health Organization 2014). This has been heavily criticized due to its innovation-hindering effects, since proving complete safety is close to impossible. Other interpretations of the precautionary principle are less riskaverse and would favour eradication efforts. Resnik (2021), for example, states that communities should decide whether the benefits of releasing genetically engineered mosquitoes trump the risks. Ostera and Gostin $(2011,931)$ emphasize the necessity for new regulatory pathways and the establishment of a new treaty body. They argue that "if the scientific evidence demonstrates significant disease reduction with low ecological risks, the precautionary principle should not impede meaningful benefits for human health." 
In conclusion, the strict interpretation of the precautionary approach falls short as a risk-mitigating principle when discussing malaria eradication. The negative impact of malaria is too substantial to never allow any risks in trying to combat it. A risk assessment, as recommended by the WHO, or a milder interpretation of the precautionary principle, takes this argument into account and should therefore be adopted.

\section{Societal Consequences}

In 2018, approximately 2.7 billion dollars was invested globally in malaria control and elimination efforts (World Health Organization 2019b). This in itself is money invested in a worthy cause. Yet, ethical issues arise when, inevitably, trade-offs have to be made when these funds are assigned. Finite resources must be divided between an expanding range of competing health programmes. For malaria, the possibilities include: socioeconomic development, insecticide use, water management, research into vaccines, drug development, or recently, focus on gene-editing technologies to modify insect vectors. It should be evaluated whether the funds that are directed towards new biotechnological techniques would not be put to better use by investing in other areas, such as socioeconomic development.

Poverty is a prime cause of malaria prevalence in sub-Saharan Africa (Degarege et al. 2019). People living in poverty do not always have the means to buy insecticides, bed nets, or drugs against malaria (Macintyre et al. 2002). Indirect reasons include badly built houses that allow Anopheles mosquitoes to enter more easily (Lindsay et al. 2003) and limited education resulting in low levels of awareness about malaria prevention and treatment (Tarimo et al. 2000; Njama et al. 2003). Reducing poverty would decrease the malaria burden but would also reduce the prevalence of other non-communicable diseases such as diabetes or cardiovascular diseases (Pullar et al. 2018). Therefore, public health measures and increased investments aimed at socioeconomic development as opposed to novel biotechnologies could be very beneficial (Tusting et al. 2013). However, eradicating malaria would reduce poverty since the disease hinders economic development (Arrow, Panosian, and Gelband 2004). Also, socioeconomic status of a country is not the only reason for a higher malaria rate; environmental conditions also play a big role (Endo and Eltahir 2016). The latest WHO report on malaria eradication states that eradication of malaria will result in a return on investment of billions of dollars (World Health Organization 2020b). Moreover, turning a blind eye to diseases while focusing on socioeconomic advancement would be unethical from a justice point of view, since access to healthcare is a fundamental right of every person.

A complex and ethically-laden technology like gene drives needs effective and robust decision-making processes. The question of who should be involved in these processes is of great importance. The National Academies of Sciences, Engineering, and Medicine (2016) describe engagement as a vital part of research and development of gene drives. The WHO also emphasizes the need for community engagement in genetically modified mosquito trials (World Health Organization 2014). Genetic modifications generally have encountered much resistance from communities. The introduction of gene drives would be even more challenging, because it is a new and complex technology that is hard to explain. This causes a so called "cognitive distance" between scientists, policymakers, regulators, and the public (Cisnetto and Barlow 2020). It is of vital importance to gain public trust in order to get acceptance. To obtain this, it is necessary to be sufficiently transparent as a company or research group (Cisnetto and Barlow 2020).

Oxitec, a British biotechnology company, has similarly developed genetically engineered mosquitoes. The company targets Aedes Aegypti mosquitoes which are vectors for diseases such as Zika and dengue fever. Their technology is based on a "self-limiting gene" that keeps female mosquitoes from surviving. The gene is non-persistent, which means that, unlike gene drives, the genes follow a Mendelian inheritance pattern (Oxitec 2020). This means that new modified mosquitoes have to be released regularly in order to keep the gene present in the ecosystem (Cisnetto and Barlow 2020). The company has carried out successful field trials in the Cayman Islands and Brazil where the Ae. aegypti population was decreased by 80 and 95 per cent, respectively (Harris et al. 2012; Carvalho et al. 2015). When Oxitec introduced their GMMs in the Cayman Islands they had approval from the government but failed to obtain consent from or even inform the local community (Resnik 2018). This led to a lot of criticism and the following field trials in Malaysia, Brazil, and Florida were conducted with ethical community engagement. It appears that when the community is effectively involved, this led to a more receptive position 
concerning the technology (Resnik 2018). When Target Malaria executed a small field trial with GMMs in a village in Burkina Faso, they engaged actively with the local community. They co-developed an acceptance model in which a reference community group was established (Target Malaria 2019). Resnik (2018) outlined common standards in guidelines used for ethical engagement with communities in his paper. These include timeliness, consent, information sharing, transparency, responsiveness, mutual understanding, respectfulness, and inclusiveness. If Target Malaria continues to engage with the communities in which further field trails will be conducted according to these standards, public trust will probably gradually be obtained which is of crucial importance for the eventual actual release of A. gambiae.

So far, only field trials have been implemented in which the GMMs were physically or ecologically contained. In these situations, the GMMs are unlikely to escape and the trials can quickly be terminated (World Health Organization 2014). The phase of actual implementation, or open-field releases, is accompanied by even more ethical complications. The mobility of mosquitoes can cause the GMMs to travel across borders. Transboundary movement issues have been tackled by, among others, the Cartagena Protocol on Biosafety (CPB). The CPB is the most important internationally ratified treaty that has an effect on the regulation of GMMs in developing countries (World Health Organization 2014). The protocol describes an Advance Informed Agreement (AIA) procedure that applies before the first intentional transboundary migration of living modified organisms (Convention on Biological Diversity 2013). However, the AIA procedure requires that the possibility of unintentional transboundary movement needs to be determined. Since this is very difficult to determine and prevent with gene drives, environmental release would not be possible (Marshall 2010). When the CPB was established, gene drives were not in the picture yet and new issues arise with this new technology. New protocols need to be established to tackle the problems around transboundary movement. Further developments in the technology, such as daisy-chain gene drives, could also decrease the threat of the gene drives spreading to non-targeted areas (Noble et al. 2019).

An issue which has yet to be addressed is the level of consent required. Community consent might become inadequate since the mosquitoes can travel around and across entire countries. Whether or not GMMs can be released in a country will most likely be decided by the national governments. However, ethical engagement with the community in places where the mosquitoes are released remains necessary. This community also needs to be accurately defined. Resnik $(2019,242)$ proposes that the community includes "people who live near enough to the proposed field trial site that their health or environment is likely to be directly and immediately impacted by the release." Non-target, neighbouring areas would be part of the public engagement. If the GMMs were then to spread farther than expected, it would be crucial that everyone had received sufficient information about the technology. Therefore, an extensive information campaign is needed in the countries where the mosquitoes might live and breed. This will be a difficult but necessary challenge in the implementation and future of the technology.

\section{Conclusion}

Prevention of human suffering by eliminating infectious diseases is a commonly accepted ethical principle that has been central in policymaking and science. It is also an ethical foundation of public health. However, there are different ways to achieve this goal, and the most ethically justified means should be used to accomplish this. Although genetically engineering an organism might be justified from a public health perspective, it raises several ethical concerns. This is especially the case when the technique causes the organism to become extinct since the risks might be too great to warrant the intervention. A detailed balance sheet must be drawn up detailing the possible favourable and adverse consequences, to enable a well informed decision about the implementation of such a measure.

The eradication of species for the sake of public health raises many ethical concerns. The fact that people have already caused the eradication of many other species and could purposefully eradicate another one just to save more human lives demands serious deliberation. The intrinsic value and moral status of a species must be taken into consideration when eradication is discussed. As A. gambiae are generally ascribed a low moral status, its eradication from the earth would not be mourned by many. Even the renowned conservationist E.O. Wilson has expressed the wish to eradicate A. gambiae (Adler 2016). 
The use of gene drives to eliminate A. gambiae is met with a lot of apprehension due to its side effects. However, the current broad-spectrum interventions against malaria possibly decrease biodiversity more than the species-specific gene drive technologies would. Nonetheless, a harm-benefit analysis must be made to determine whether releasing genetically engineered mosquitoes into the wild would be beneficial. Then, a decision must be made regarding acceptable risks. Taking into account that the eradication of malaria would save hundreds of thousands of lives each year, it would be immoral to reject this technique altogether.

It seems that policymakers have to make a decision about whether to invest in new biotechnologies to combat malaria or focus on socioeconomic development. However, eradicating malaria will also have a positive impact on a nation's socioeconomic status. A not-forprofit organization such as Target Malaria can assist in this by developing and providing GMMs, complementary to current efforts against malaria. However, it is vital for such an organization to be sufficiently transparent and to address public trust. Community engagement will be of great importance to achieve this.

The eventual release of the GMMs will be regulated by national governments. They have to form an agreement due to possible transboundary movement of the mosquitoes. The Cartagena Protocol on Biosafety provides a guideline for this, but new protocols will be needed to tackle this issue. Obtaining public consent for a technology implemented on a large scale will represent a very difficult hurdle in the practical application of this technology. A first step towards public engagement will be to make sure that the public has sufficient information about the technology.

Acknowledgements We would like to thank Dr. Philippe Van den Steen for providing feedback on the manuscript.

\section{References}

Adler, J. 2016. Kill all the mosquitoes?! Smithsonian Magazine, June. https://www.smithsonianmag.com/innovation/kill-allmosquitos-180959069/?page=2. Accessed December 29, 2019.

AMCA. 2019. American Mosquito Control Association. https://www.mosquito.org/. Accessed December 29, 2019.

Arrow, K.J., C. Panosian and H. Gelband. 2004. The human and economic burden of Malaria. In Saving Lives, Buying Time: Economics of Malaria Drugs in an Age of Resistance Library of Congress Cataloging-in-Publication Data, edited by K.J. Arrow, C. Panosian, and H. Gelband, 168-196. Washington (DC): National Academies Press (US).

BBC. 2014. Animal ethics: Moral status of animals. British Broadcasting Corporation. http://www.bbc.co. uk/ethics/animals/rights/moralstatus_1.shtml. Accessed April 7, 2020.

Brossard, D., P. Belluck, F. Gould and C.D. Wirz. 2019. Promises and perils of gene drives: Navigating the communication of complex, post-normal science. Proceedings of the National Academy of Sciences of the United States of America 116(16): 7692-7697.

Callaway, E. 2015. Mosquitoes engineered to pass down genes that would wipe out their species. Nature News. https://doi. org/10.1038/nature.2015.18974.

Caminade, C., S. Kovats, J. Rocklov, et al. 2014. Impact of climate change on global malaria distribution. Proceedings of the National Academy of Sciences of the United States of America 111(9): 3286-3291.

Carrington, D. 2019. What is biodiversity and why does it matter to us? The Guardian, March 12. https://www.theguardian. com/news/2018/mar/12/what-is-biodiversity-and-why-doesit-matter-to-us. Accessed December 30, 2021.

Carvalho, D.O., A.R. McKemey, L. Garziera, et al. 2015. Suppression of a field population of Aedes aegypti in Brazil by sustained release of transgenic male mosquitoes. PLoS Neglected Tropical Diseases 9(7): e0003864.

CDC. 2016. History of smallpox. https://www.cdc. gov/smallpox/history/history.html. Accessed December 5, 2019.

. 2019. Guinea worm disease eradication program. https:/www.cdc.gov/parasites/guineaworm/gwep.html. Accessed April 9, 2020.

Chen, I., S.E. Clarke, R. Gosling, et al. 2016. 'Asymptomatic' malaria: A chronic and debilitating infection that should be treated. PLoS Medicine 13(1): e1001942.

Cisnetto, V., and J. Barlow. 2020. The development of complex and controversial innovations. Genetically modified mosquitoes for malaria eradication. Research Policy 49(3): 103917.

CITES. 2011. The CITES Species Appendix-Animals checklist of CITES species. https://www.cites.org/eng/disc/species.php. Accessed April 7, 2020.

Collins, C.M., J.A.S. Bonds, M.M. Quinlan, and J.D. Mumford. 2018. Effects of the removal or reduction in density of the malaria mosquito, Anopheles gambiae s.1., on interacting predators and competitors in local ecosystems. Medical and Veterinary Entomology 33(1): 1-15.

Convention on Biological Diversity. 2013. Text of the Cartagena Protocol on Biosafety. The Biosafety Clearing-House (BCH). Secretariat of the Convention on Biological Diversity.

Convention on Biological Diversity Conference of the Parties. 2012. COP 11 Decision XI/11-New and emerging issues relating to the conservation and sustainable use of biodiversity.

Degarege, A., K. Fennie, D. Degarege, S. Chennupati and P. Madhivanan. 2019. Improving socioeconomic status may reduce the burden of malaria in sub Saharan Africa: A systematic review and meta-analysis. PLOS ONE 14(1): $\mathrm{e} 0211205$.

DeGrazia, D. 2008. Moral status as a matter of degree? Southern Journal of Philosophy 46(2): 181-198. 
Endo, N., and E.A.B. Eltahir. 2016. Environmental determinants of malaria transmission in African villages. Malaria Journal 15(1): 1-11.

Esvelt, K.M., A.L. Smidler, F. Catteruccia and G.M. Church. 2014. Concerning RNA-guided gene drives for the alteration of wild populations. eLife 3: 1-21.

European Commission. 2000. Communication from the Commission on the Precautionary Principle Commission of the European Communities.

—. 2017. EU Biodiversity Strategy for 2030. https://ec. europa.eu/environment/nature/biodiversity/strategy/index_ en.htm. Accessed April 6, 2020.

-2019. Why do we need to protect biodiversity?https://ec. europa.eu/environment/nature/biodiversity/intro/index_en. htm. Accessed April 14, 2020.

Fang, J. 2010. Ecology: A world without mosquitoes. Nature 466(7305): 432-434.

Feachem, R.G.A., I. Chen, O. Akbari, et al. 2019. Malaria eradication within a generation: Ambitious, achievable, and necessary. The Lancet 394(10203): 1056-1112.

Gascon, C., T.M. Brooks, T. Contreras-Macbeath, et al. 2015. The importance and benefits of species. Current Biology 25(10): 431-438.

Gates, B. 2019. Test tube mosquitoes might help us beat malaria GateNotes. https://www.gatesnotes.com/Health/Test-tubemosquitoes-might-help-us-beat-malaria. Accessed December 7, 2019.

Hammond, A.M. and R. Galizi. 2017. Gene drives to fight malaria: Current state and future directions. Pathogens and Global Health 111(8): 412-423.

Hammond, A.M., K. Kyrou, M. Bruttini, et al. 2017. The creation and selection of mutations resistant to a gene drive over multiple generations in the malaria mosquito. PLoS Genetics 13(10): e1007039.

Harris, A.F., A.R. McKemey, D. Nimmo, et al. 2012. Successful suppression of a field mosquito population by sustained release of engineered male mosquitoes. Nature Biotechnology 30(9): 828-830.

Hay, S.I., C.A. Guerra, A.J. Tatem, A.M. Noor and R.W. Snow. 2004. The global distribution and population at risk of malaria: Past, present, and future. Lancet Infectious Diseases 4(6): 327-336.

Hayirli, T.C., and P.F. Martelli. 2019. Gene drives as a response to infection and resistance. Infection and Drug Resistance 12: 229-234.

IUCN. 2020. Biodiversity and protected areas. https://www.iucn. org/commissions/world-commission-protected-areas/ourwork/biodiversity-and-protected-areas. Accessed April 6, 2020.

Kyrou, K., A.M. Hammond, R. Galizi, et al. 2018. A CRISPRCas9 gene drive targeting doublesex causes complete population suppression in caged Anopheles gambiae mosquitoes. Nature biotechnology 36(11): 1062-1066.

Lindsay, S.W., M. Jawara, K. Paine, et al. 2003. Changes in house design reduce exposure to malaria mosquitoes. Tropical Medicine and International Health 8(6): 512-517.

Louca, S., P.M. Shih, M.W. Pennell, et al. 2018. Bacterial diversification through geological time. Nature Ecology and Evolution 2(9): 1458-1467.
Macintyre, K., J. Keating, S. Sosler, et al. 2002. Examining the determinants of mosquito-avoidance practices in two Kenyan cities. Malaria Journal 1(1): 1-14.

Marshall, J.M. 2010. The Cartagena Protocol and genetically modified mosquitoes. Nature Biotechnology 28(9): 896-897.

Murphy, B., and C. Jansen. 2010. Risk analysis on the Australian release of Aedes aegypti (L.) (Diptera: Culicidae) containing Wolbachia CSIRO.

Naegeli, H., J.L. Bresson, T. Dalmay, et al. 2020. Adequacy and sufficiency evaluation of existing EFSA guidelines for the molecular characterisation, environmental risk assessment and post-market environmental monitoring of genetically modified insects containing engineered gene drives. EFSA Journal 18(11): e06297.

National Academies of Sciences, Engineering, and Medicine. 2016. Gene drives on the horizon: Advancing science, navigating uncertainty, and aligning research with public values. National Academies Press. https://doi.org/10.17226 $/ 23405$.

Neves, M.P., and C. Druml. 2017. Ethical implications of fighting malaria with CRISPR/Cas9. BMJ Global Health 2(3): 9-11.

Njama, D., G. Dorsey, D. Guwatudde, et al. 2003. Urban malaria: Primary caregivers' knowledge, attitudes, practices and predictors of malaria incidence in a cohort of Ugandan children. Tropical Medicine and International Health 8(8): 685-692.

Nkya, T.E., I. Akhouayri, W. Kisinza and J.P. David. 2013. Impact of environment on mosquito response to pyrethroid insecticides: Facts, evidences and prospects. Insect Biochemistry and Molecular Biology. 43(4): 407-416.

Noble, C., J. Min, J. Olejarz, et al. 2019. Daisy-chain gene drives for the alteration of local populations. Proceedings of the National Academy of Sciences of the United States of America 116(17): 8275-8282.

Ojala, M., and R. Lidskog. 2011. What lies beneath the surface? A case study of citizens' moral reasoning with regard to biodiversity. Environmental Values 20(2): 217-237.

Open Philanthropy. 2017. Target Malaria - Gene drives for malaria control open philanthropy. https://www.openphilanthropy. org/focus/scientific-research/miscellaneous/target-malariageneral-support. Accessed April 21, 2020.

Ostera, G.R., and L.O. Gostin. 2011. Biosafety concerns involving genetically modified mosquitoes to combat malaria and dengue in developing countries. JAMA 305(9): 930-931.

Oxitec. 2020. Our technology. https://www.oxitec.com/en/ourtechnology. Accessed April 16, 2020.

Pandas International. 2020. Panda reserves Pandas International. https:/www.pandasinternational.org/panda-reserves/. Accessed April 6, 2020.

Pearce, N. 2004. Public health and the precautionary principle. In Precautionary Principle: Protecting Public Health, The Environment, and the Future of our Children, edited by M. Martuzzi, and J.A. Tickner, 49-62. World Health Organization.

Piperaki, E.-T. 2018. Malaria eradication in the European world: Historical perspective and imminent threats. In Towards malaria elimination-A leap forward, edited by $\mathrm{S}$. Manguin, and and V. Dev. IntechOpen. https://doi. org/10.5772/intechopen.76435.

Pugh, J. 2016. Driven to extinction? The ethics of eradicating mosquitoes with gene-drive technologies. Journal of Medical Ethics 42(9): 578-581. 
Pullar, J., L. Allen, N. Townsend, et al. 2018. The impact of poverty reduction and development interventions on noncommunicable diseases and their behavioural risk factors in low and lower-middle income countries: A systematic review. PLoS ONE 13(2): e0193378..

Regan, T. 2004. The case for animal rights. University of California Press.

Resnik, D.B. 2018. Ethics of community engagement in field trials of genetically modified mosquitoes. Developing World Bioethics 18(2): 135-143.

- 2019. Two unresolved issues in community engagement for field trials of genetically modified mosquitoes. Pathogens and Global Health 113(5): 238-245.

- 2021. Precautionary reasoning in environmental and public health policy. Cham: Springer International Publishing (The International Library of Bioethics).

Rosenfeld, J.S. 2002. Functional redundancy in ecology and conservation. Oikos 98(1): 156-162.

Roser, M., and H. Ritchie. 2019. Malaria Our World in Data. https://ourworldindata.org/malaria\#malaria-was-commonacross-half-the-world-since-then-it-has-been-eliminated-inmany-regions. Accessed December 5, 2019.

Save the Rhino. 2020. Save the Rhino International | Rhino Conservation Charity Save the Rhino. https://www. savetherhino.org/what-we-do/protecting-rhinos/. Accessed April 6, 2020.

Scudellari, M. 2019. Self-destructing mosquitoes and sterilized rodents: The promise of gene drives. Nature 571(7764): 160-162.

Singer, P. 2016. Are insects conscious? Project Syndicate. https://www.project-syndicate.org/commentary/are-insectsconscious-by-peter-singer-2016-05?barrier=accesspaylog. Accessed January 4, 2020.

van der Sluijs, J.P., and N.S. Vaage. 2016. Pollinators and global good security: The need for holistic global stewardship. Food Ethics 1(1): 75-91.

Sodhi, N.S., and P.R. Ehrlich. 2010. Conservation biology for all. Oxford University Press.

Soulé, M.E. 1985. What is conservation biology? BioScience 35(11): 727-734.

Target Malaria. 2019. Target Malaria proceeded with a smallscale release of genetically modified sterile male mosquitoes in Bana, a village in Burkina Faso Target Malaria. https://targetmalaria.org/target-malaria-proceeded-with-asmall-scale-release-of-genetically-modified-sterile-malemosquitoes-in-bana-a-village-in-burkina-faso/. Accessed April 27, 2020.

- 2020. Our work Target Malaria. https://targetmalaria. org/our-work/. Accessed January 4, 2020.

Tarimo, D.S., G.K. Lwihula, J.N. Minjas and I.C. Bygbjerg. 2000. Mothers' perceptions and knowledge on childhood malaria in the holendemic Kibaha district, Tanzania: Implications for malaria control and the IMCI strategy. Tropical Medicine and International Health 5(3): 179-184.
Tusting, L.S., B. Willey, H. Lucas, et al. 2013. Socioeconomic development as an intervention against malaria: A systematic review and meta-analysis. The Lancet 382(9896): 963-972.

UN. 2020. Convention on Biological Diversity UN. https://www. cbd.int/. Accessed April 6, 2020.

Unckless, R.L., A.G. Clark and P.W. Messer. 2017. Evolution of resistance against CRISPR/Cas9 gene drive. Genetics 205(2): 827-841.

Weinbauer, M., and F. Rassoulzadegan. 2007. Extinction of microbes: Evidence and potential consequences. Endangered Species Research 3: 205-215.

World Health Organization. 2014. Guidance framework for testing of genetically modified mosquitoes. https://www.who. int/tdr/publications/year/2014/guide-fmrk-gm-mosquit/en/. Accessed April 27, 2020.

. 2017. A framework for malaria elimination Geneva: World Health Organization. https://www.who. int/publications-detail-redirect/9789241511988. Accessed November 1, 2021.

- 2019a. Global targets WHO. World Health Organization. https://www.who.int/malaria/areas/global_targets/en/. Accessed January 4, 2020.

_ 2019b. World malaria report. https://www.who. int/news-room/fact-sheets/detail/malaria. Accessed November 27, 2019.

. 2020a. Malaria WHO. https://www.who.int/newsroom/fact-sheets/detail/malaria. Accessed March 9, 2020.

- 2020b. Malaria eradication: Benefits, future scenarios and feasibility. https://www.who.int/publicationsdetail/malaria-eradication-benefits-future-scenariosfeasibility. Accessed May 15, 2020.

- 2020c. World Malaria Report: 20 years of global progress and challenges World Health. https://www.who. int/publications/i/item/9789240015791. Accessed November 1, 2020.

- 2021. WHO recommends groundbreaking malaria vaccine for children at risk. https://www.who.int/news/item/0610-2021-who-recommends-groundbreaking-malariavaccine-for-children-at-risk. Accessed November 1, 2021.

World Animal Protection. 2018. World animal protection. https://www.worldanimalprotection.org/. Accessed April 7, 2020.

World Wildlife Fund (WWF). 2015. How does biodiversity loss affect me and everyone else? Biodiversity. https://wwf.panda. org/our_work/biodiversity/biodiversity_and_you/. Accessed April 6, 2020.

Xu, X.R.S., E.A. Bulger, V.M. Gantz, et al. 2020. Active genetic neutralizing elements for halting or deleting gene drives. Molecular Cell 80(2): 246-262.

Publisher's note Springer Nature remains neutral with regard to jurisdictional claims in published maps and institutional affiliations. 